\title{
Uric acid, lung function and COPD: a causal link is unlikely
}

\author{
Seif O Shaheen
}

\author{
Happy the man who has been able to learn \\ the causes of things-Virgil
}

In epidemiology we strive to identify the root causes of disease, in the hope that this will enable us to devise preventive strategies. Observational studies constitute our 'bread and butter' and, in carrying out such studies, we find associations and make judgements on whether they have arisen through chance, bias or confounding, before considering possible causal interpretations. Confounding is the bane of an epidemiologist's life because, however hard we try to rule it out as a possible explanation for a link between exposure and disease in observational studies, it is nigh on impossible to do so with total confidence. While we do our best to take potential confounders 'out of the equation' in our analyses, usually by controlling for them in multivariable regression models, if confounders have been measured imprecisely there will still be residual confounding, and other confounders may not have been measured (some may be unknown) and therefore cannot be controlled for.

The gold standard study design for obtaining unconfounded evidence of causality is the randomised controlled trial (RCT), because randomisation should lead to even distribution of confounders between treatment arms. However, sometimes trials are not ethical to conduct and, even if feasible, large trials are very expensive and time-consuming. In recent years a welcome addition to the epidemiologist's armoury for strengthening causal inference in observational studies has been Mendelian randomisation (MR), an approach championed by George Davey Smith and colleagues, ${ }^{1}$ which can be regarded as a 'natural RCT'. MR is a form of instrumental variable analysis, whereby a genetic variant can be used as a proxy for a specific exposure to test whether that exposure is likely to be causally related to an outcome. ${ }^{1}$ A fundamental principle underpinning $\mathrm{MR}$ is that, as genotypes are randomly allocated during meiosis, associations between genetic variants and

Correspondence to Professor Seif O Shaheen, Centre for Primary Care and Public Health, Blizard Institute, Barts and the London School of Medicine and Dentistry, London E1 2AT, UK; s.shaheen@qmul.ac.uk disease are not generally susceptible to confounding by lifestyle factors, which is a major strength of this approach. Similarly, as genotype cannot be influenced by disease, associations detected by MR cannot be explained by reverse causation. In the context of respiratory health and disease, MR has been used to demonstrate that, while elevated serum/plasma $\mathrm{C}$ reactive protein (CRP) is associated with lower lung function and an increased risk of COPD in observational analyses, genetically elevated CRP is not, suggesting that CRP is not causally related to these outcomes. $^{2} 3$ However, MR cannot be used to test all aetiological hypotheses; it is simply not possible in the absence of suitable polymorphisms for modifiable exposures of interest. ${ }^{1}$

Oxidative stress is thought to play an important role in the pathogenesis of $\mathrm{COPD}^{4}$ and impaired lung function, ${ }^{5}$ and epidemiological studies have suggested that a diet rich in antioxidants might counteract the effects of smoking and reduce the risk of COPD. ${ }^{6}$ Endogenous antioxidants are also thought to play an important defensive role in the lungs, and uric acid (UA), which has antioxidant properties, is present in high concentrations in the epithelial lining fluid (ELF) of the airways. ${ }^{78}$ A limited number of epidemiological studies have therefore investigated whether higher concentrations of serum UA are associated with higher levels of lung function, but results have been contradictory. Cross-sectional studies of adults have reported both positive ${ }^{9}$ and inverse $^{10}$ associations with lung function; a cohort study found that higher serum UA concentrations predicted a lower incidence of COPD, especially in smokers. ${ }^{11}$ However, interpretation of these studies requires caution, for a number of reasons. Aside from the problem of potential confounding, reverse causation (whereby the presence of respiratory inflammation generates an increased antioxidant response) cannot be discounted in the cross-sectional studies, and serum UA concentrations may not be a good proxy for ELF concentrations. ${ }^{8}$ Also, a high UA status could be viewed as a doubleedged sword; while it may have beneficial antioxidant effects, it may also, paradoxically, have pro-oxidant and proinflammatory properties, ${ }^{12} 13$ as well as increase the risk of gout. In a previous editorial, it was suggested that MR could potentially provide more robust evidence, either supporting or refuting a causal association (protective or deleterious) between UA and lung function and COPD. ${ }^{14}$ Indeed, this approach has been used extensively to investigate the relation between UA and a wide range of non-respiratory health outcomes. Genomewide association studies have identified multiple gene variants that predict serum $\mathrm{UA}^{15}$ and which can therefore be used as unconfounded proxy measures of lifetime UA status. A recent review of the evidence from 107 MR studies, covering 56 unique health outcomes (including anthropometric, cardiovascular, metabolic, neurocognitive and renal outcomes), concluded that gout was the only condition for which convincing evidence was presented for a causal link with elevated serum UA. ${ }^{16}$

Kobylecki et $a l^{17}$ have carried out the first MR study to determine whether plasma UA is causally linked to lung function and COPD. One strength of this rigorous study is the large sample sizecomprising over 100000 adults from two independent general population samples in Denmark; genetic instruments usually only explain a very small proportion of the variance in the exposure of interest, and many previous MR studies of UA and other health outcomes have been underpowered to detect small or modest causal effects. ${ }^{16}$ Use of a genetic risk allele score, based on two variants known to predict higher plasma UA concentrations, will have increased statistical power further. ${ }^{18}$ Kobylecki and colleagues ${ }^{17}$ first analysed the association between plasma UA and respiratory outcomes in the conventional way, and found that, after controlling for many potential confounders, higher UA concentrations were associated with lower lung function and increased risk of symptoms and COPD, in both samples. However, when they used MR, they found that the genetic risk score was not associated with these outcomes, suggesting no causal association. Likely explanations for the associations between measured plasma UA and respiratory outcomes include residual or unmeasured confounding, and/ or reverse causation. Other strengths of the study relate to three core assumptions which need to be satisfied for a genetic variant to be a valid instrument for causal inference. First, the authors confirmed that the genetic risk score was strongly associated with the exposure of interest (plasma UA concentrations) in the study population. Second, the genetic score was not associated with measured confounders of the exposure-outcome associations, 
thus making it less likely that the score was confounded by unmeasured confounders; confounding by population substructure (ie, by genetic ancestry, whereby genotype frequency and prevalence of the phenotypes of interest vary by ethnic subgroups within the population) is unlikely as all individuals were of Danish descent. Third, given what is known about the function of the genes involved, the genetic variants being used as instrumental variables are likely to influence the disease outcomes under study only through the exposure of interest (plasma UA); in other words, 'horizontal pleiotropy', whereby the gene variants influence the outcome through pathways that do not include the exposure of interest, is less likely to be occurring (although this is hard to prove). ${ }^{19}$ It has been suggested that negative MR results may be more reliable than positive results $^{20}$; had the authors found that the genetic risk score was associated with the outcomes of interest, there are recently developed analytical approaches and sensitivity analyses that could have been used to try to strengthen causal inference further (eg, to test whether the 'no pleiotropy' assumption had been violated) and obtain even more robust conclusions. ${ }^{19}$

In summary, MR is potentially a powerful tool to strengthen causal inference in suitably large studies (tens of thousands of individuals), and this latest study provides the most robust test to date of the UA-lung function/COPD hypothesis; the results are certainly likely to be more reliable, and closer to 'the truth', than those obtained from conventional observational analyses. These new data provide rather convincing evidence that UA is unlikely to be causally linked to lung function and COPD, and it would therefore seem appropriate to draw a line under this hypothesis in terms of further investigation. Importantly, we can say, with a high degree of confidence, that higher UA status is not beneficial for lung function and COPD risk. From a public health perspective, this is perhaps just as well, as intervention strategies designed to raise blood UA concentrations would be problematic, given the increased risk of gout.

Funding The authors have not declared a specific grant for this research from any funding agency in the public, commercial or not-for-profit sectors.

Competing interests None declared.

Provenance and peer review Commissioned; externally peer reviewed.

(C) Article author(s) (or their employer(s) unless otherwise stated in the text of the article) 2018. All rights reserved. No commercial use is permitted unless otherwise expressly granted.

\section{A) Check for updates}

To cite Shaheen SO. Thorax 2018;73:697-698.

Accepted 5 March 2018

Published Online First 23 March 2018

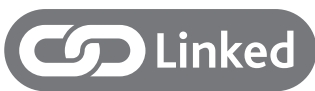

- http://dx.doi.org/10.1136/thoraxjnl-2017-210273

Thorax 2018;73:697-698.

doi:10.1136/thoraxjnl-2017-211230

\section{REFERENCES}

1 Smith GD, Ebrahim S. 'Mendelian randomization': can genetic epidemiology contribute to understanding environmental determinants of disease? Int J Epidemiol 2003;32:1-22.

2 Bolton CE, Schumacher W, Cockcroft JR, et al. The CRP genotype, serum levels and lung function in men: the Caerphilly Prospective Study. Clin Sci 2011;120:347-55.

3 Dahl M, Vestbo J, Zacho J, et al. C reactive protein and chronic obstructive pulmonary disease: a Mendelian randomisation approach. Thorax 2011;66:197-204.

4 Ciencewicki J, Trivedi S, Kleeberger SR. Oxidants and the pathogenesis of lung diseases. J Allergy Clin Immunol 2008;122:456-68.
5 Schünemann HJ, Muti P, Freudenheim JL, et al. Oxidative stress and lung function. Am J Epidemiol 1997; 146:939-48.

6 Varraso R, Shaheen SO. Could a healthy diet attenuate COPD risk in smokers? Thorax 2017;72:491-2.

7 Kelly FJ, Blomberg A, Frew A, et al. Antioxidant kinetics in lung lavage fluid following exposure of humans to nitrogen dioxide. Am J Respir Crit Care Med 1996;154:1700-5.

8 van $\operatorname{der}$ Vliet $A, O^{\prime}$ Neill $C A$, Cross $C E$, et al. Determination of low-molecular-mass antioxidant concentrations in human respiratory tract lining fluids. Am J Physiol 1999;276:L289-L296.

9 Song JU, Hwang J, Ahn JK. Serum uric acid is positively associated with pulmonary function in Korean health screening examinees. Mod Rheumatol 2017;27:1057-65.

10 Aida Y, Shibata Y, Osaka D, et al. The relationship between serum uric acid and spirometric values in participants in a health check: the Takahata study. Int $J$ Med Sci 2011;8:470-8.

11 Horsfall LJ, Nazareth I, Petersen I. Serum uric acid and the risk of respiratory disease: a population-based cohort study. Thorax 2014;69:1021-6.

12 So A, Thorens B. Uric acid transport and disease. J Clin Invest 2010;120:1791-9.

13 Lyngdoh T, Marques-Vidal P, Paccaud F, et al. Elevated serum uric acid is associated with high circulating inflammatory cytokines in the population-based Colaus study. PLoS One 2011;6:e19901.

14 Shaheen SO. Antioxidants and respiratory disease: the uric acid paradox. Thorax 2014;69:978-9.

15 Kolz M, Johnson T, Sanna S, et al. Meta-analysis of 28,141 individuals identifies common variants within five new loci that influence uric acid concentrations. PLoS Genet 2009;5:e1000504.

16 Li X, Meng $X$, Timofeeva M, et al. Serum uric acid levels and multiple health outcomes: umbrella review of evidence from observational studies, randomised controlled trials, and Mendelian randomisation studies. BMJ 2017;357:;2376.

17 Kobylecki CJ, Vedel-Krogh S, Afzal S, et al. Plasma urate, lung function and chronic obstructive pulmonary disease: a Mendelian randomisation study in 114 979 individuals from the general population. Thorax 2018;73:748-57.

18 Burgess S. Sample size and power calculations in Mendelian randomization with a single instrumental variable and a binary outcome. Int J Epidemiol 2014;43:922-9.

19 Zheng J, Baird D, Borges MC, et al. Recent Developments in Mendelian Randomization Studies. Curr Epidemiol Rep 2017:4:330-45.

20 VanderWeele TJ, Tchetgen Tchetgen EJ, Cornelis $\mathrm{M}$, et al. Methodological challenges in mendelian randomization. Epidemiology 2014;25:427-35. 